Paper presented at the first Topical Meeting on Fusion Reactor Materials, Miami Beach, Florida, January 28-31, 1979.

\title{
SPATIAL VARIATION IN VOID VOLUME DURING CHARGED PARTICLE BOMBARDMENT - THE EFFECTS OF INJECTED INTERSTITIALS*
}

E. H. LEE, L. K. MANSUR, and M. H. YOO Oak Ridge National Laboratory Oak Ridge, Tennessee 37830

\section{ABSTRACT}

Experimental observations of the void volume at several depths along the range of $4 \mathrm{MeV} \mathrm{Ni}$ ions in 316 stainless steel are reported. The specimens were first preconditioned by neutron irradiation at temperatures of 450 and $584^{\circ} \mathrm{C}$ to $\mathrm{fluences}$ of approximately $8 \times 10^{26} \mathrm{n} / \mathrm{m}^{-2}$. The void volume after ion bombardment to $60 \mathrm{dpa}$ at the peak damage depth is significantly lower at the peak damage depth than in the region between that and the free surface. The ratio of the step height to void volume at the depth of peak energy deposition between regions masked from and exposed to the beam is strongly dependent on bombardment temperature. The reduction of void volume near the peak damage depth is larger for the $584^{\circ} \mathrm{C}$ than for the $450^{\circ} \mathrm{C}$ preconditioned material. These observations are consisțent with recent theoretical results which account for the injection of the bombarding ions as self-interstitials. The theory necessary to understand the effect is developed.

\section{MASTER}

$*$ Research sponsored by the Division of Materials Sciences and Office of Reactor Research and Technology, U.S. Department of Energy under contract W-7405-eng-26 with the Union Carbide Corporation. 
Paper presented at the First Topical Meeting on Fusion Reactor Materials, Miami Beach, Florida, January 28-31, 1979.

\title{
SPATIAL VARIATION IN VOID VOLUME DURING CHARGED PARTICLE BOMBARDMENT - THE EFFECTS OF INJECTED INTERSTITIALS*
}

\author{
E. H. LEE, L. K. MANSUR, and M. H. YOO \\ Oak Ridge National Laboratory \\ Oak Ridge, Tennessee $\mathbf{3 7 8 3 0}$
}

\section{INTRODUCTION}

To characterize the effects of radiation in potential materials for fusion reactors, use is being made of presently available irradiation devices: iission reactors, accelerator-driven neutron sources, and charged particle accelerators. The highest displacement rates, $10^{3}-10^{4}$ times the rates of projected fusion reactors, are only available from charged particle accelerators. The corresponding contraction in the time scale $t$ achieve a given dose is a great benefit and is largely responsible for th. widespread use of accelerators for radiation effects studies. However, this benefit is only obtained at the expense of extensive theoretical analys is to transform the results to projections for reactor conditions.

The problem areas may be separated into three, which we characterize as rate effects, spatial effects, and impurity and phase stability effects. This last topic concerns the behavior of alloying elemenis in terms of point defect trapping characteristics, relative mobilities and propensity to segregate, and formation of new phases under various conditions. The topic is very broad and has been extensively examined at a recent conference whose proceedings are available [1]. Simpler rate effects arise from the very large differences in point defect generation rates. The most widely recognized consequence of this difference is a shift to higher temperature of the regime of swelling 
with higher generation rate. The effect has been described in a number of publications. See Refs. [2] and [3] and the references cited therein.

The large increase in point defect generation rate with charged particles is primarily a consequence of the large stopping cross sections of high energy charged particles as compared to high energy neutrons. The rapid stopping of the bombarding particle during heavy-ion bombardment is also responsible for the short range of heavy ions as well as for a pronounced spatial dependence of point defect generation along the range. In addition, the ions injected as interstitials during self-ion bombardment may produce a perturbation in the void volume profile." The resulting void volume can be very nonuniform in contrast to the nearly uniform swelling during neutron irradiation. The results of theoretical analysis of spatial effects are described elsewhere [4-7]. The purpose here is to present experimental results which provide the first documentation of a substantial depletion of void volume due to the injection of self-interstitials. The theory necessary to understand the effect is described.

\section{THEORY}

The rate equations ${ }^{\circ}$ wich govern the vacancy and interstitial concentrations during charged particle bombardment may be written ${ }^{\dagger}$

$$
\begin{aligned}
& \nabla \cdot\left(D_{v} \nabla C_{v}\right)+G_{v}-R C_{v} C_{i}-K_{v} C_{v}=\frac{\partial C_{v}}{\partial t} \\
& \nabla \cdot\left(D_{i} \nabla C_{i}\right)+G_{i}-R C_{v} C_{i}-K_{i} C_{i}=\frac{\partial C_{i}}{\partial t}
\end{aligned}
$$

\footnotetext{
*Actually, injected interstitials increase the swelling in general by the addition of extra atoms. Any injected interstitials not absorbed at voids must increase the swelling. However, the effect to be described here is the reduction in void volume, that quantity obtained by electron microscopy observation, by injected interstitials.

tFor purposes of clarity these equations are written in the form applicable to pure materials, i.e., ignoring point defect trapping.
} 
The G's describe the generation rates of vacancies and interstitials (subscripts $v$ and $i$ ) per unit time per unit volume. $G_{v}=G\left(1-\varepsilon_{v}\right)+G_{T}$ where $G$ is the displacement produced-generation rate and $\varepsilon_{v}$ is the fraction retained in vacancy clusters, here modeled as vacancy loops. $G_{T}$ is the thermal generation rate, where $G_{T}=\sum_{j} G_{T}^{j}=D_{v} C_{v}^{e} \sum \xi^{j} S_{v}^{j}$. Here $D_{v}=D_{v}^{0} \exp \left(-E_{v}^{m} / k T\right)$ is the vacancy diffusion coefficient where $D_{v}^{0}$ is a constant, $E_{v}^{m}$ is the vacancy migration energy, $k$ is Boltzmann's constant and $T$ is absolute temperature. $c_{v}^{e}$ is the bulk thermal concentration of free vacancies, and $\xi^{j}$ is the ratio of thermal vacancies at: sink type $j$ to that in the bulk. For voids $\xi^{V}=\exp \left[\left(\frac{2 \gamma}{r_{V}}-P_{g}\right) \Omega / k T\right]$, where $\gamma$ is the surface tension, $r_{V}$ is the void radius, $\Omega$ is the atomic volume, and $P_{g}$ is the pressure of any contained gas; for dislocation loops $\xi^{\ell}=\exp \left[ \pm\left(\gamma_{f}+E_{\ell}\right) a^{2} / k T\right]$, where $\gamma_{f}$ is the stacking fault energy, $E_{\ell}$ is the loop elastic energy, and a is a lattice dimension; for dislocations $\xi_{v}^{d}=1$. $s_{v}^{j}$ is the strength for vacancies of the sink of type $j$; for voids $s_{v}^{v}=4 \pi \int r_{v} z_{v}^{v}\left(r_{v}\right) n\left(r_{v}\right) d r_{v},=4 \pi r_{v} \bar{z}_{v}^{N_{v}}$ if average values are used, where $z_{v}^{v}\left(r_{v}\right)$ is the capture efficiency for vacancies of a void of radius $r_{V}, n\left(r_{V}\right) d r_{V}$ is the number of voids per unit volume bstween radi $r_{v}$ and $r_{v}+d r_{v} . \bar{r}_{v}$ is the average void radius and $N_{V}$ is the tota' number of voids per unit volume and $\bar{z}_{v}^{v}$ the average capture efficiency. For dislocations $s_{v}^{d}=z_{v}^{d} L$ where $z_{v}^{d}$ is the capture efficiency of a dislocation for vacancies and $L$ is the dislocation density. Dislocation loops may be modeled as effective spherical sinks where the form of their sink efficiency follows that for voids, or as equivalent lengths of dislocation line where the form follows that for dislocation lines. The diffusion coefficient and sink strengths for interstitials are obtained from the expressions above for vacancies by replacing $v$ with $i$.

The terms $R C_{i} C_{v}, K_{v} C_{v}$ and $K_{i} C_{i}$ describe respectively the loss of free vacancies and interstitials by mutual recombination, and loss of free vacancies 
and free interstitials to sinks. $C_{v}$ and $C_{i}$ are respectively the concentrations of free vacancies and interstitials per unit volume. $R=4 \pi r_{0}\left(D_{i}+D_{v}\right)$ is the coefficient of recombination where $r_{0}$ denotes the radius of the recombination volume. $K_{V}$ and $K_{i}$ are the loss rates to all continuum sinks per point defect for vacancies and interstitials respectively. Thus $k_{v}=\sum_{j} k_{v}^{j}$ and $K_{\mathbf{i}}=\sum_{\mathbf{j}} k_{\mathbf{i}}^{\mathbf{j}}$ where j represents each sink type such as void, dislocation; precipitate, and grain boundary. These coefficients are simply related to the sinks strengths given above as $K_{v}^{j}=D_{v} S_{v}^{j}$ and $K_{i}^{j}=D_{i} S_{i}^{j}$.

The first term in eqs. (1) and (2) describes diffusional leakage caused by the proximity of sinks such as free surfaces which are modeled as discrete, i.e., not included among the continuum internal sinks represented by $k_{v}$ and $K_{i}$. Depletion of the swelling caused by the presence of surfaces can be pronounced in regions near the surfaces, at high temperatures or with very low internal sink strengths. The effect is minor for the experimental conditions evaluated in this paper at the depths of interest, i.e., $20.4 \mu \mathrm{m}[6,7]$. Similarly the first terms of eqs. (1) and (2) leads to an additional depletion of swelling during heavy ion bombardment by the fact that the point defect generation rate is spatially dependent with a pronounced peak. For example, the peak occurs near $0.7 \mu \mathrm{m}$ in a $4 \mathrm{MeV} \mathrm{Ni}$ bombardment of stainless steel. Because there is a peak, diffusional spreading and smoothing of the point defect concentrations and of the swelling occurs. Under certain conditions the depletion in swelling resulting from this spreading can be pronounced as compared to a neutron irradiation where this effect is absent. The effect is exposed in more detail in Ref. [7]. The source of the effect to be highlighted here is the contribution of injected self-ions to the interstitial generation rate. In eq. (2) the total interstitial generation rate is given by 
$G_{i}=G\left(1+\varepsilon_{i}\right)=G+G_{i}$ where $\varepsilon_{i}$ is the fraction of interstitials injected by the bombarding beam.

Figure 1 shows the effective point defect generation rate profile as well as the self-ion injection profile for $4 \mathrm{MeV}$ nickel bombardment of nickel as calculated using the EDEP-1 program of Manning and Mueller [8]. This figure illustrates the spatial dependence of $\varepsilon_{i}$ in relation to $G_{i}$ described in the previous paragraph, and shows that $\varepsilon_{i}$ is of order $10^{-4}-10^{-3}$.

Brailsford and Mansur [9] evaluated analytically the effect of injected interstitials. With $\varepsilon_{i}$ in this range they predicted a depletion in void growth rate ranging from a few percent to tens of percent depending on the temperature, for the conditiors and parameter range investigated. They also pointed out that the depletion is sensitive to vacancy migration energy slightly raising the migration energy causes a significant increase in the depletion of void growth rate due to injected interstitials. The calculations of Yoo [6] incorporating the depth dependence of interstitial injection confirm these results. This can be understood based on the fraction of point defects recombining in the matrix. A low vacancy migration energy increases vacancy mobility, decreases vacancy concentration and lowers the fraction of point defects recombining. When most defects go to sinks the injected interstitials comprise only a small fraction of the defects absorbed at sinks. However, a number of interstitials equal to the number of injected interstitials may never recombine since there is no corresponding vacancy produced. Therefore, injected interstitials comprise a larger fraction of point defects absorbed at sinks whenever the fraction of point defects recombining is increased. A number of physical conditions may result in a large fraction of defects recombining. In particular, materials with a high vacancy migration anergy, at low temperatures, with low biases or with low internal sink strengths should exhibit significant depletion of void volume caused by injected interstitials. Reference [7] may be consulted for additional exposition of these effects. In this paper, theory 
and experiment are combined to identify a strong reduction of void volume caused by injected interstitials in nickel-ion-bombarded 316 stainless steel.

Figure 2 shows the calculated ratio of void growth rate with injected interstitials to thest without injected interstitials as a function of the strength of internal sinks for vacancies, for several different values of bias. It is seen that for low sink strengths and biases of order $10 \%$, conditions which are applicable to stainless steels, that injected interstitials are expected to produce a significant reduction in void growth rate particularly at low sink strengths

\section{EXPERIMENTAL}

The experiment to be described examines the void volume of specimens which have been preconditioned by neutron irradiation and subsequently ion boinbarded. The neutron exposure was approximately $8 \times 10^{26} \mathrm{n} / \mathrm{m}^{2}$ at temperatures of 450 and $584^{\circ} \mathrm{C}$. These conditions resulted in substantial swelling and microstructural evolution prior to the ion bombardment. Such preconditioning treatment offers two major advantages which facilitate direct comparisons with the theory. Firstly, the ion bombardment produces mainly growth of an initially spatially uniform distribution of voids. This obviates the need for a theoretical calculation of void nucleation. Although cheoretical models exist for void nucleation, their results are extremely sensitive to imprecisely known materials and defect parameters such as surface tension and impurity-point defect trapping parameters. Thus, the analysis rests on the established theory of void growth [10]. Secondly, the fact that different specimens have been preconditioned at two widely different temperatures adds an important dimension to the value of this data for comparison with theory. The material preconditioned at $450^{\circ} \mathrm{C}$ contains after ion bombardment a total void plus dislocation strength of $5.9 \times 10^{14} \mathrm{~m}^{-2}$ while the corresponding value for the material preconditioned at $585^{\circ} \mathrm{C}$ is $1.6 \times 10^{14} \mathrm{~m}^{-2}$. As shown in the previous 
section, the theory predicts a significantly greater suppression of void growth by injected interstitials for the higher temperature treatment with this difference of nearly a factor of four in sink strength.

\subsection{Procedure}

The specimens used in this experiment were $3-\mathrm{mm}$ disks of SA 316 stainless steel from heat $M 2783$ cut from tubes irradiated in EBR-11. Prior to nickel ion bombardment, the specimens were polished mechanically and electrolytically which minimized damage to the surface. The specimens were bombarded using the $4 \mathrm{MeV} \mathrm{Ni}{ }^{++}$ions.

Following bombardment, the surface step between the bombarded surface and a region masked from the beam was determined using a calibrated profilometer capable of detecting steps as small as $50 \AA$. Specimens were then sectioned to desired depths for TEM using the recently developed multisectioning technique described elsewhere [11].

\section{RESULTS}

Figure 3 shows the relationship between the step height and the peak damage void volume (SH/PV ratio) for specimens ion bombarded to 60 dpa at four temperatures. For specimens preconditioned at $450^{\circ} \mathrm{C}$ the $\mathrm{SH} / \mathrm{PV}$ ratio decreased with increasing temperature from $\sim 30 \mathrm{~nm} / 1 \%$ at $550^{\circ} \mathrm{C}$ to $15 \mathrm{~nm} / 1 \%$ at $650^{\circ} \mathrm{C}$. At higher temperatures the ratios could not be determined reliably because of the large uncertainties involved in measuring steps $<20 \mathrm{~nm}$. For specimens preconditioned at $584^{\circ} \mathrm{C}$, the ratio was even higher at lower temperatures and decreased more rapidly with increasing temperature.

Figure 4 shows the void volume directly determined by transmission electron microscopy as a function of depth below the ion entry surface using the multisectioning technique on single specimens. A typical set of migrographs is shown in Figure 5 . In both cases void volume is significantly higher at 
about $450 \mathrm{~nm}$ than at the approximately $700 \mathrm{~nm}$ predicted by

calculation of swelling based on the energy deposition profile of Figure 1 when injected interstitials are not taken into account $[6,7]$.

\section{SUMMARY AND DISCUSSION}

The experimental results show three important features.

1. The void volume peaks at a depth of $2450 \mathrm{~nm}$ from the surface rather than at $\sim 700 \mathrm{~nm}$ where the peak in energy deposition occurs.

2. The void volume at the depth near the peak in energy deposition is lower in relation to the void volume at $\sim 450 \mathrm{~nm}$ for the material preconditioned at $584^{\circ} \mathrm{C}$ as compared to that preconditioned at $450^{\circ} \mathrm{C}$.

3. The step height/peak void volume ratio, where this void volume is measured near the peak in energy deposition, decreases with increasing temperature of ion bombardment. This variation is more pronounced for the material preconditioned at $584^{\circ} \mathrm{C}$ as compared to that preconditioned at $450^{\circ} \mathrm{C}$.

These results can be explained as a manifestation of the swelling suppression caused by injected interstitials. With respect to item 1, our calculations confirm that the depth of maximum void volume which occurs at $\sim 700 \mathrm{~nm}$ with no injected interstitials can be shifted as far forward as $450 \mathrm{~nm}$ by including injected interstitials. For the sink strengths observed in these specimens this requires an effective vacancy migration energy of about $1.5 \mathrm{eV}$. While this is higher than the range of accepted values for nickel, $1-1.4 \mathrm{eV}$, it is plausible that in this alloy the vacancy migration energy is higher. For example, vacancy trapping on impurities or alloying elements raises the effective vacancy diffusion coefficient [12].

Item 2 is understood in terms of Fig. 2. It shows that the effect of injected interstitials should be more pronounced for the material preconditioned

*Al though the middle and bottom micrographs may appear more similar than the results plotted in. Fig. 4, it should be remembered that Fig. 4 shows the additional void volume produced by ion bombardment. 
at $584^{\circ} \mathrm{C}$, as compared to the material preconditioned at $450^{\circ} \mathrm{C}$, since the void plus dislocation sink strengths are respectively $7.2 \times 10^{13}$ and $7.6 \times 10^{14}$ before ion bombardment and $1.6 \times 10^{14} \mathrm{~m}^{-2}$ and $5.9 \times 10^{14}$ near the peak damage depths after ion bombardment.

The variability of step height/peak void volume ratio with temperature, item 3 , can also be explained based on injected interstitials. Temperature and depth dependent calculations, examples of which are given in Ref. [7], show that the suppression of void volume by injected interstitials is more pronounced at low temperature. This follows since the fraction of displacement produced defects migrating to sinks is lower at lower temperatures because of enhanced recombination. The injected interstitials affect void growth most where they are injected at the highest rate - from Fig. I this occurs at $2800 \mathrm{~nm}$, near the peak damage region. Since the step height measures the swelling integrated along the entire depth, its value will be reduced less than the void volume at peak damage depth by injected interstitials. The larger step height to peak void volume ratio in material pre-conditioned at $585^{\circ} \mathrm{C}$ results from the lower sink strength in this material at all ion bombardment temperatures. The theory shows that injected interstitials are important in suppressing void growth where point defect recombination is important. Thus the effect is expected to be least in high swelling alloys. This may help explain why Whitley et al. [13] do not observe evidence of void growth suppression by injected interstitials for pure nickel. The effect may also be masked by implantation of gas prior to or during bombardment since void nucleation is strongly enhanced by injected gases. In addition, the use of material which has not been preconditioned complicates the picture. The analysis must then deal with nucleation and growth of interstitial-loops and void nucleation as well as void growth. While injected interstitials suppress void nucleation directly, they erihance interstitial loop nucleation and hence enhance void nucleation indirectly by providing sinks with a larger capture efficiency for interstitials in the same 
spatial region. These considerations may help explain why evidence for the effect of injected interstitials in suppressing void volume is not observed in non-preconditioned 316 stainless steel [11].

\section{REFERENCES}

[1] Proceedings of the Workshop on Solute Segregation and Phase Stability during Irradiation, Nov. 1-3, 1978, Gatlinburg, Tennessee, to be published in Joumal of Muclear Materials.

[2] N. H. Packan, K. Farrell, and J. O. Stiegler, J. Mucl. Muter. 78 (1978) 143.

[3] L. K. Mansur, J. Nucl. Mater. 78 (1978) 156.

[4] M. H. Yoo and L. K. Mansur, J. Nucl. Mater. 62 (1976) 282.

[5] M. H. Yoo, J. Nucl. Mater. 68 (1977) 193.

[6] M. H. Yoo, J. Nucl. Mater. 79 (1979)! 135.

[7] L. K. Mansur and M. H. Yoo, this conference.

[8] 1. Manning and G. P. Mueller, Comp. Phys. Comm. 7 (1974) 85.

[9] A. D. Brailsford and L. K. Mansur, J. Nucl. Mater. 71 (1977) 110.

[10] L. K. Mansur, NucZ. TechnoZ. 40 (1978) 5.

[11] E. H. Lee and A. F. Rowcliffe, Miorostructural Science, Vol. 7 (1979) to be published.

[12] L. K. Mansur and M. H. Yoo, J. Hucl. Mater. 74 (1978) 22ชิ ; s9e also L. K. Mansur in Ref. [1].

[13j J. B. Whitley, G. L. Kulcinski, and P. Wilkes, this conference. 


\section{Figure Captions}

Fig. I The $4 \mathrm{MeV} \mathrm{Ni}$ defect generation rate and injected ion deposition rate profiles, as a function of the depth below the surface.

Fig. 2 The relative void growth rate as a function of the sink strength for three values of the bias.

Fig. 3 The ratio of step height to measured void volume at the peak damage depth for SA 316 stainless steel preconditioned to $8 \times 10^{26} \mathrm{n} \cdot \mathrm{m}^{-2}$ at 450 and $584^{\circ} \mathrm{i}$ and ion irradiated to $60 \mathrm{dpa}$ at four temperatures.

Fig. 4 The measured void volume with depth for SA 316 stainless steel preconditioned to $8 \times 10^{26} \mathrm{n} \cdot \mathrm{m}^{-2}$ at 450 and $584^{\circ} \mathrm{C}$ and ion irradiated to $60 \mathrm{dpa}$ at 600 and $625^{\circ} \mathrm{C}$.

Fig. 5 The microstructures of SA 316 stainless steel preconditioned to $8 \times 10^{22} \mathrm{n} \cdot \mathrm{m}^{-2}$ at 450 and $584^{\circ} \mathrm{C}$ (top two). The botiom four micrographs corresponding to Fig. 4 show void microstructures at approximately the peak clamage depth and at 0.6 of this depth. 
G, DEFECT

GENERATION RATE $\left(10^{-3} \mathrm{dpa} / \mathrm{s}\right)$

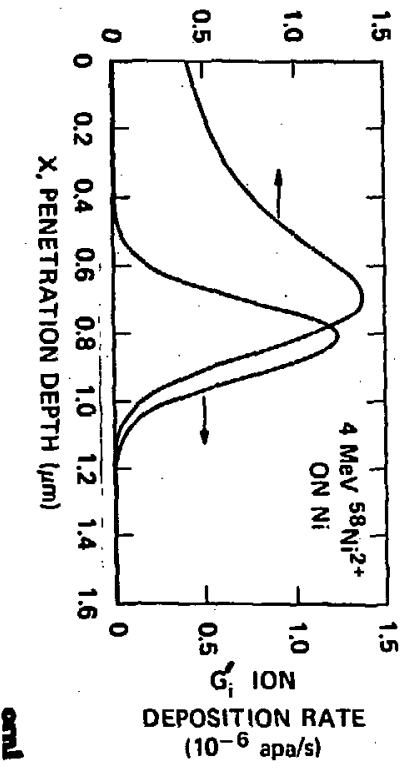




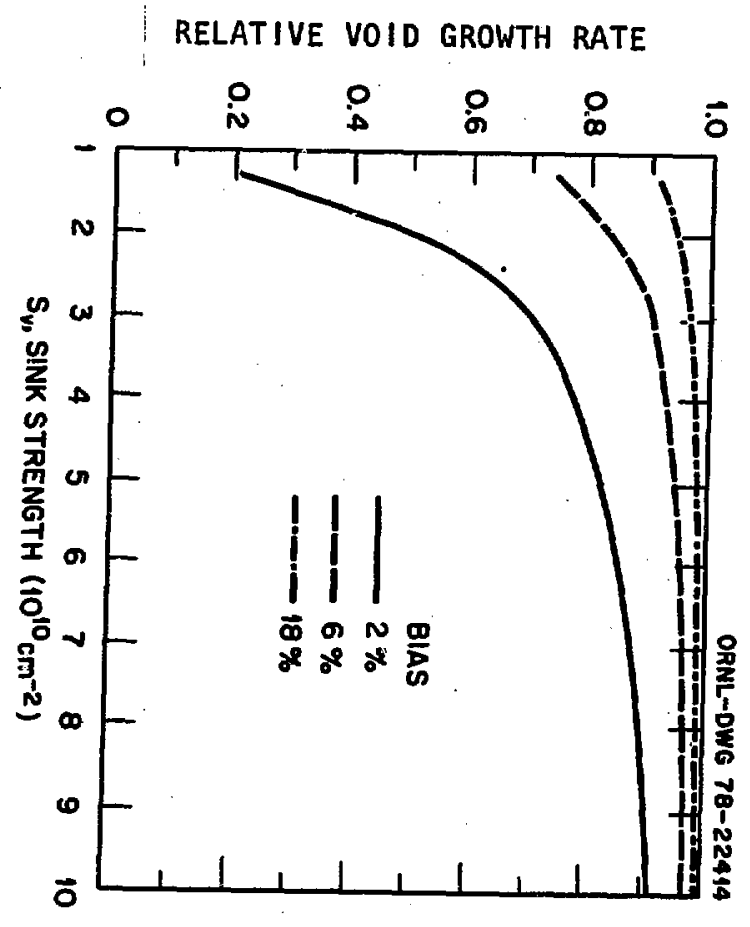




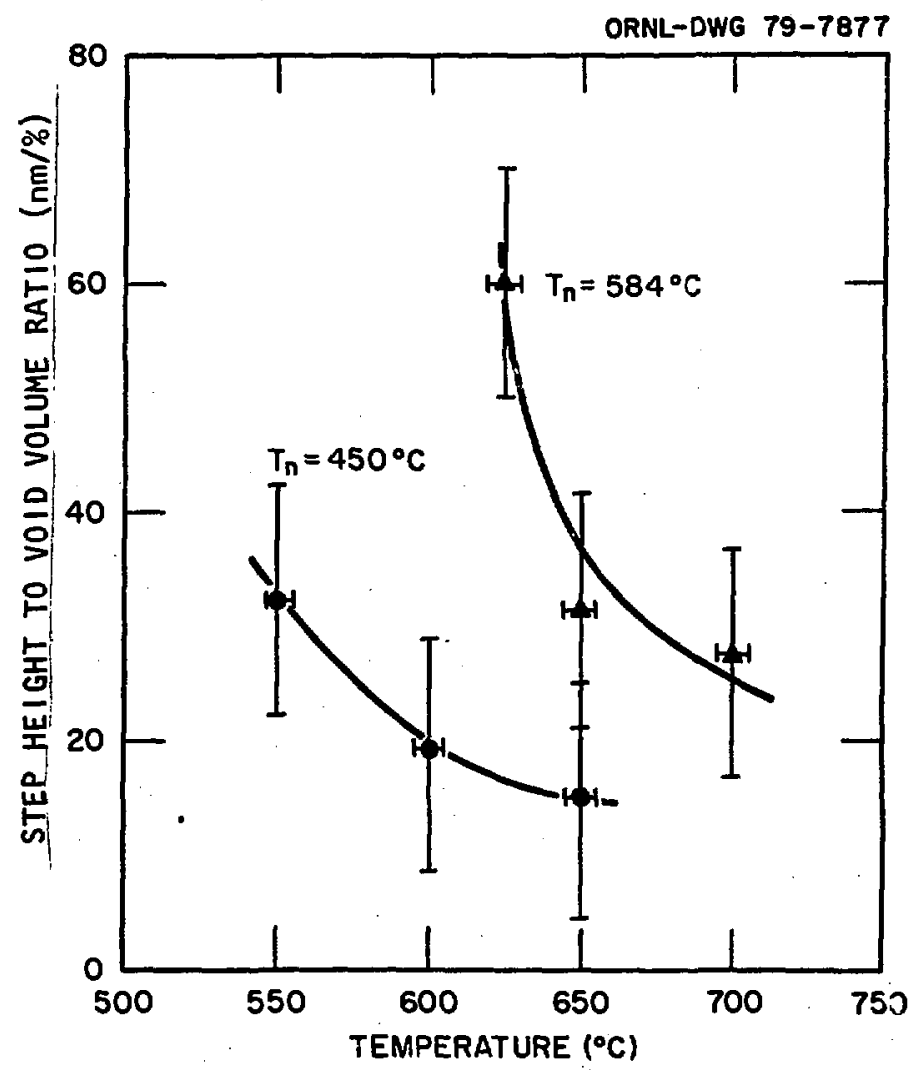




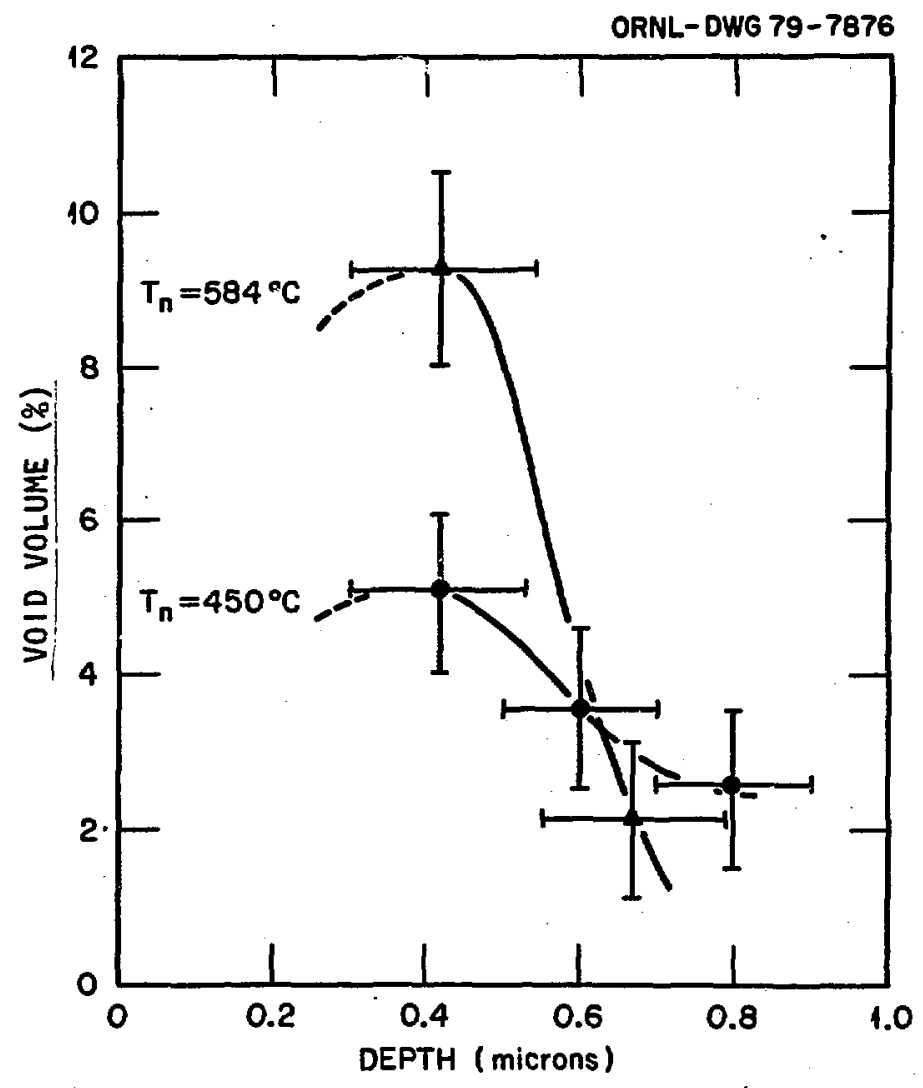



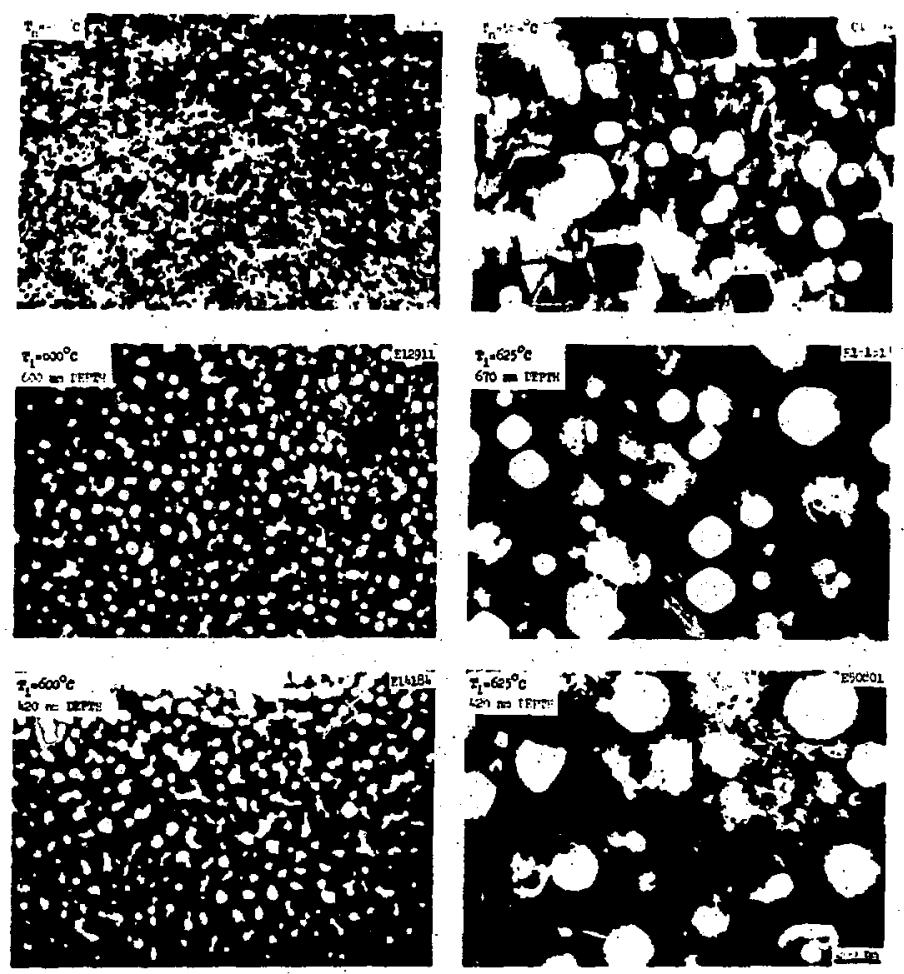

Fig. 5 